\title{
Upregulation of macrophage migration inhibitory factor promotes tumor metastasis and correlates with poor prognosis of pancreatic ductal adenocarcinoma
}

\author{
DONG WANG ${ }^{1,2^{*}}$, RUIZHI WANG ${ }^{1,2^{*}}$, ANPEI HUANG ${ }^{1}$, ZENG FANG $^{1}$, \\ KEBING WANG $^{1}$, MEIFANG HE ${ }^{1}$, JIN-TANG XIA ${ }^{3}$ and WEN LI ${ }^{1}$ \\ ${ }^{1}$ Laboratory of General Surgery and ${ }^{2}$ Division of Laboratory Medicine, \\ The First Affiliated Hospital, Sun Yat-sen University, Guangzhou, Guangdong 510080; \\ ${ }^{3}$ Department of General Surgery, The Third Affiliated Hospital, Guangzhou Medical University, \\ Guangzhou, Guangdong 510150, P.R. China
}

Received January 31, 2018; Accepted September 7, 2018

DOI: $10.3892 /$ or.2018.6703

\begin{abstract}
Macrophage migration inhibitory factor (MIF) is a pro-inflammatory cytokine that serves important roles in cancer. MIF overexpression is frequently observed in numerous human cancer types, including pancreatic carcinoma. However, the prognostic value and function of MIF in pancreatic ductal adenocarcinoma (PDAC) have not been fully elucidated. In the present study, upregulation of MIF expression in PDAC tissue compared with adjacent normal tissue was observed. Furthermore, MIF overexpression was identified to be significantly associated with poor survival rates in patients with PDAC. Multivariate Cox regression analysis confirmed that MIF was an independent risk factor for poor survival. Functional analyses demonstrated that MIF knockdown significantly inhibited the proliferation and invasion of pancreatic cancer cells in vitro compared with control cells. IN addition, mechanistic investigations revealed that silencing MIF leads to inhibition of AKT serine/threonine kinase and extracellular-signal-regulated kinase activation, and suppression of cyclin D1 and matrix metalloproteinase-2 expression, which may suppress tumor proliferation and invasion. These results highlight the importance of MIF overexpression in
\end{abstract}

Correspondence to: Dr Wen Li, Laboratory of General Surgery, The First Affiliated Hospital, Sun Yat-sen University, 58 Zhongshan Road II, Guangzhou, Guangdong 510080, P.R. China

E-mail: wenli28@163.com

Dr Jin-Tang Xia, Department of General Surgery, The Third Affiliated Hospital, Guangzhou Medical University, 63 Duobao Road, Guangzhou, Guangdong 510150, P.R. China

E-mail: 13xia@163.com

${ }^{*}$ Contributed equally

Key words: macrophage migration inhibitory factor, metastasis, overexpression, pancreatic ductal adenocarcinoma, survival period
PDAC aggressiveness, and indicate that MIF may be a potential therapeutic target for pancreatic cancer.

\section{Introduction}

Pancreatic carcinoma is a common type of cancer associated with high mortality rates and is the fourth leading cause of cancer-associated mortality globally (1). Pancreatic ductal adenocarcinoma (PDAC) is the most common type of pancreatic cancer. The 5-year overall survival rate for patients with PDAC is $\sim 5 \%$ and median survival time is $<6$ months (2). Surgical removal of the tumor is the most effective and preferred therapy (3). However, PDAC is characterized by invasive growth and early metastasis, often manifesting in advanced clinical stage at presentation and rapid postoperative recurrence, and only one fifth of patients with PDAC are diagnosed early enough to be candidates for surgical resection (4). Furthermore, due to the frequent recurrence of PDAC, the median survival time even among surgically resected cases is $<2$ years (1). Therefore, an understanding of the mechanisms of PDAC metastasis and the identification of the factors involved in early metastasis are required to improve treatment and disease outcomes in PDAC.

PDAC is associated with significant intra- and peritumorial inflammation (5). Chronic pancreatitis is a risk factor for PDAC, and new-onset pancreatitis is a common symptom of PDAC. Upregulation of inflammation-associated signaling modulates PDAC progression and therapeutic resistance by inducing proliferation and metastasis, and by suppressing apoptosis $(6,7)$. Macrophage migration inhibitory factor (MIF) is a pleiotropic inflammatory cytokine that is associated with carcinogenesis (8). Through autocrine or paracrine signaling, MIF interacts with cluster of differentiation (CD)74, its primary receptor, and $\mathrm{C}-\mathrm{X}-\mathrm{C}$ chemokine receptor type 4 , its co-receptor, to activate the AKT serine/threonine kinase (AKT) and extracellular-signal-regulated kinase (ERK) pathways $(9,10)$. Previous studies from different groups have identified an increased expression level and tumor-promoting functions of MIF in PDAC (11-14). MIF knockdown inhibits 
ERK1/2 and AKT phosphorylation, and upregulates p53 expression, in turn leading to cell cycle arrest and apoptosis in pancreatic cancer cells $(11,12)$. Recently, Yang et al (13) reported a novel signaling pathway whereby MIF upregulates miR-301b, which subsequently targets nuclear receptor subfamily 3 group $\mathrm{C}$ member 2 . Inhibition of this signaling axis may reduce metastasis and prolong survival in a mouse model of PDAC (13). Furthermore, MIF induces the epithelial to mesenchymal transition and invasion of PDAC cells $(13,14)$. Nevertheless, the role of MIF in pancreatic cancer is not clearly defined.

In the present study, MIF were demonstrated to enhance cyclin D1 and matrix metalloproteinase (MMP)-2 expression by activating AKT and ERK signaling; subsequently promoting metastasis of PDAC cells. We also show that upregulation of MIF is a frequent event in PDAC, and correlates with unfavorable prognosis in pancreatic cancer.

\section{Materials and methods}

PDAC tissue samples. The present study was approved by the Human Research Ethics Committees of the First Affiliated Hospital of Sun Yat-sen University (approval no. 201515; Guangzhou, China), according to the Declaration of Helsinki. All of patients enrolled in the present study provided written informed consent.

Human PDAC tissues were collected from 85 patients who underwent resection at the First Affiliated Hospital of Sun Yat-sen University between 2003 and 2007. Patients did not receive any local or systemic chemotherapeutic treatments prior to the surgery. Tumor histopathology was independently classified according to the World Health Organization Classification of Tumors by two pathologists (15). All patients were followed postoperatively to assess survival outcomes. The relevant characteristics of patients are listed in Table I.

Immunohistochemistry (IHC). Formalin-fixed paraffin-embedded PDAC tissues were cut into $5 \mu \mathrm{m}$ sections, processed for antigen retrieval by pressure cooking in $10 \mathrm{mM}$ citrate buffer (pH6.0) and blocked with UltraCruz ${ }^{\circledR}$ Blocking reagent (cat. no. sc-516214; Santa Cruz Biotechnology, Inc., Dallas, TX, USA) at room temperature for $1 \mathrm{~h}$, followed by incubation at $4^{\circ} \mathrm{C}$ overnight with rabbit polyclonal antibody against human MIF (cat. no. sc-20121; Santa Cruz Biotechnology, Inc.). Immunostaining was performed with the ChemMate DAKO EnVision Detection kit for Peroxidase/DAB/Rabbit/Mouse (cat. no. K 5007; Dako; Agilent Technologies, Inc., Santa Clara, CA, USA) according to the manufacturer's protocol, which resulted in a brown-colored precipitate at the antigen site.

MIF in PDAC tissues was evaluated under a light microscope at $\mathrm{x} 400$ magnification. For each specimen, five images of representative areas were acquired, and a total of 1,000 tumor cells were counted. IHC scoring was performed according to a modified Histo-score (H-score) (16), which includes an assessment of the fraction of positive cells and the intensity of staining. The intensity was assigned a score of $0-3$, representing no staining for 0 , weak staining for 1 , moderate staining for 2 , and strong staining for 3 . The fraction score was based on the proportion of positively stained cells $(0-100 \%)$. The intensity and fraction scores were multiplied to obtain an H-score that ranged between 0 and 3 , and represented the expression level of MIF protein.

RNA oligoribonucleotides, tumor cell, lines and transfection. Small interfering RNA (siRNA) duplexes that target the human MIF mRNA (si-MIF) were designed using BLOCK-iT ${ }^{\mathrm{TM}}$ RNAi Designer software (Thermo Fisher Scientific, Inc., Waltham, MA, USA) and purchased from Guangzhou RiboBio Co., Ltd. (Guangzhou, China). Negative control RNA duplexes (NC) for siRNA were not homologous to any known human sequence. The nucleotide sequences of si-MIF and NC are as follows: si-MIF sense, 5'-GGGUCUACAUCAACUAUUAdT dT-3' and antisense, 5'-UAAUAGUAGUUGUAGACCCdT dT-3; NC-sense, 5'-UUCUCCGAACGUGUCACGUdTdT-3' and antisense, 5'-ACGUGACACGUUCGGAGAGAAdTdT-3'.

Human panc-1 and Bxpc-3 pancreatic cancer cell lines were obtained from the Cell Bank of Type Culture Collection of Chinese Academy of Sciences (Shanghai, China) and maintained in a humidified $5 \% \mathrm{CO}_{2}$ incubator at $37^{\circ} \mathrm{C}$. Human panc-1 pancreatic cancer cell lines were cultured in Dulbecco's modified Eagle's medium (DMEM; Invitrogen; Thermo Fisher Scientific, Inc.) and Bxpc-3 pancreatic cancer cells were cultured in RPMI-1640 (RPMI; Gibco; Thermo Fisher Scientific, Inc.) supplemented with $10 \%$ fetal bovine serum (FBS; HyClone; GE Healthcare Life Sciences, Logan, UT, USA). RNA oligonucleotides were transfected using Lipofectamine RNAiMAX (Invitrogen; Thermo Fisher Scientific, Inc.). A total of $5 \times 10^{4}$ cells/well were transfected with $50 \mathrm{nM}$ RNA in a 24-well plate.

$R N A$ isolation, reverse transcription-semi-quantitative polymerase chain reaction ( $R T-s q P C R)$ and $R T-q P C R$. Total RNA was isolated from cells or frozen tumor tissues using TRIzol reagent (Invitrogen; Thermo Fisher Scientific, Inc.), according to the manufacturer's protocol. RNA concentration and quality were evaluated according to spectrometric determination at 260 and $280 \mathrm{~nm}$. A total of $2 \mu \mathrm{g}$ of total RNA was subjected to DNase I digestion (Fermentas; Thermo Fisher Scientific, Inc.), followed by RT using Moloney murine leukemia virus reverse transcriptase (Promega Corporation, Madison, WI, USA) at $42^{\circ} \mathrm{C}$ for $1 \mathrm{~h}$ followed by termination at $75^{\circ} \mathrm{C}$ for $5 \mathrm{~min}$. Then, mRNA levels of MIF, cyclin D1, and MMP-2 were analyzed by RT-sqPCR (for cDNA of PDAC tissues and cell lines) or by SYBR-Green (cat. no. A25742; Applied Biosystems; Thermo Fisher Scientific, Inc.) RT-qPCR (for cDNA of cell lines). The specific primers used to amplify the MIF, cyclin D1, or MMP-2 genes and the housekeeping GAPDH gene were as follows: MIF forward, 5'-GCAGAACCGCTCCTACAG CA-3' and reverse, 5'-GGCTCTTAGGCGAAGGTGGA-3'; Cyclin D1 forward, 5'-GCTGCTCCTGGTGAACAAGC-3' and reverse, 5'-CACAGAGGGCAACGAAGGTC-3'; MMP-2 forward, 5'-AGAGTGCATGAACCAACCAG-3' and reverse, 5'-TGTTCAGGTATTGCATGTGCT-3'; GAPDH forward, 5'-GAGTCAACGGATTTGGTCGT-3' and reverse, 5'-GAC AAGCTTCCCGTTCTCAG-3'. GAPDH expression served as an internal control. For RT-sqPCR, the cDNA was amplified as follows: $94^{\circ} \mathrm{C}$ for $30 \mathrm{sec}, 58^{\circ} \mathrm{C}$ for $30 \mathrm{sec}$ and $72^{\circ} \mathrm{C}$ for $30 \mathrm{sec}$ for 28 cycles; and a final extension at $72^{\circ} \mathrm{C}$ for $5 \mathrm{~min}$. The products were resolved on a $1.5 \%$ agarose gel and visualized 
Table I. Association between MIF Expression and clinical features.

\begin{tabular}{|c|c|c|c|c|}
\hline \multirow[b]{2}{*}{ Variables } & \multirow[b]{2}{*}{ Case no. } & \multicolumn{2}{|c|}{ MIF } & \multirow[b]{2}{*}{ P-value ${ }^{a}$} \\
\hline & & Low & High & \\
\hline Sex & & & & 0.156 \\
\hline Male & 51 & 22 & 29 & \\
\hline Female & 34 & 20 & 14 & \\
\hline Age, years & & & & $0.023^{\mathrm{b}}$ \\
\hline$>50$ & 69 & 30 & 39 & \\
\hline$\leq 50$ & 16 & 12 & 4 & \\
\hline Tumor size, $\mathrm{cm}$ & & & & 0.614 \\
\hline$>3$ & 48 & 22 & 26 & \\
\hline$\leq 3$ & 37 & 18 & 17 & \\
\hline Differentiation & & & & 0.892 \\
\hline Well and moderate & 52 & 26 & 26 & \\
\hline Poor & 33 & 16 & 17 & \\
\hline TNM & & & & 0.332 \\
\hline III/IV & 36 & 20 & 16 & \\
\hline $\mathrm{I} / \mathrm{II}$ & 49 & 22 & 27 & \\
\hline Lymphatic spread & & & & $0.036^{\mathrm{b}}$ \\
\hline No & 49 & 29 & 20 & \\
\hline Yes & 36 & 13 & 23 & \\
\hline Hepatic metastasis & & & & 0.750 \\
\hline No & 66 & 32 & 34 & \\
\hline Yes & 19 & 10 & 9 & \\
\hline Serum CEA, ng/ml & & & & $0.022^{\mathrm{b}}$ \\
\hline$\geq 10$ & 49 & 19 & 30 & \\
\hline$<10$ & 36 & 23 & 13 & \\
\hline
\end{tabular}

Analysis was performed on 85 cases. The MIF level was examined

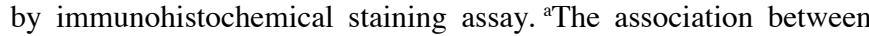
MIF level and clinical features of patients with PDAC was analyzed by chi-square test. ${ }^{b} \mathrm{P}<0.05$, statistically significant difference. MIF, Macrophage migration inhibitory factor; TNM, tumor node metastasis; CEA, carcinoembryonic antigen.

using ethidium bromide staining with GeneSnap software (version 1.2.0; Syngene, Frederick, MD, USA). RT-qPCR was performed using a 7800 fast real-time PCR system (Applied Biosystems; Thermo Fisher Scientific, Inc.) with the following thermocycling conditions: $94^{\circ} \mathrm{C}$ for $30 \mathrm{sec}, 60^{\circ} \mathrm{C}$ for $30 \mathrm{sec}$ and $72^{\circ} \mathrm{C}$ for $30 \mathrm{sec}$. The gene expression levels were normalized to expression of GAPDH to calculate the $2^{-\Delta \Delta \mathrm{Cq}}$ value (17).

Immunoblotting assay. Proteins from cells or frozen tumor tissues were extracted with RIPA buffer (Invitrogen; Thermo Fisher Scientific, Inc.) and quantified using a bicinchoninic acid assay. The total protein ( $20 \mu \mathrm{g} / \mathrm{lane})$ was separated on a $12 \%$ polyacrylamide gel, and transferred to a methanol-activated PVDF membrane (EMD Millipore, Billerica, MA, USA). The membrane was blocked in Tris-buffered saline-Tween-20 (TBST) containing 5\% bovine serum albumin (Guangzhou
Jetway Biotech Co., Ltd., Guangzhou, China) at room temperature for $1 \mathrm{~h}$, then subsequently immunoblotted with primary rabbit polyclonal antibodies against MIF (cat. no. sc-20121; Santa Cruz Biotechnology, Inc.), cyclin D1 (cat. no. 2978; Cell Signaling Technology, Inc., Beverly, MA, USA), MMP-2 (cat. no. 40994; Cell Signaling Technology, Inc.) or GAPDH (cat. no. BM1623; Wuhan Boster Biological Technology, Ltd., Wuhan, China) at $4^{\circ} \mathrm{C}$ overnight and then with the secondary antibody (anti-rabbit IgG horseradish peroxidase-conjugated; cat. no. 7074; Cell Signaling Technology, Inc.) at room temperature for $1 \mathrm{~h}$. All the primary antibodies were diluted at a ratio of 1:1,000 and the secondary antibody was diluted at a ratio of 1:5,000. Protein bands were visualized using Clarity Western ECL substrate (Bio-Rad Laboratories, CA, USA). The intensity of each band was densitometrically quantified using ImageJ software (version 1.0; National Institutes of Health, Bethesda, MD, USA).

Cell cycle analysis. Cell cycle analyses were performed using a detergent containing hypotonic solution (Krishan's reagent) containing $10 \mu \mathrm{g} / \mathrm{ml}$ propidium iodide (Sigma-Aldrich; Merck KGaA, Darmstadt, Germany) according to the manufacturer's protocol and fluorescence-activated cell sorting (FACS) with a Gallios flow cytometry system (Beckman Coulter, Inc., Brea, CA, USA) assay. Data were analyzed using Kaluza software (version 1.5a; Beckman Coulter, Inc.). Nuclear debris and overlapping nuclei were gated out.

In vitro tumor cell invasion assay. Tumor cell invasion was analyzed in 24-well Boyden chambers with $8-\mu \mathrm{m}$ pore size polycarbonate membranes (Corning Incorporated, Corning, NY, USA). The membranes were coated with $60 \mu \mathrm{g}$ of Matrigel (cat. no. 3432-005-01; R\&D Systems, Inc., Minneapolis, MN, USA) to form the matrix barrier. Pancreatic cancer cells transfected with NC or si-MIF were resuspended in $100 \mu \mathrm{l}$ serum-free DMEM $36 \mathrm{~h}$ post-transfection, and were added to the upper compartments of the chambers. The lower compartments were filled with $600 \mu 1$ DMEM or RPMI-1640 with $10 \%$ FBS. Following an incubation at $37^{\circ} \mathrm{C}$ for $24 \mathrm{~h}$, the cells remaining on the upper surfaces of the membrane that had not invaded through the matrix were removed. The invaded cells on the lower surfaces of the membrane were fixed with $100 \%$ methanol at room temperature for $10 \mathrm{~min}$, stained with $0.1 \%$ crystal violet at room temperature for $15 \mathrm{~min}$ and counted under a light microscope at $\mathrm{x} 400$.

Statistical analysis. Associations between MIF expression and clinicopathological features were examined using the chi-square test. Overall survival was calculated as the duration between the date of tumor resection and the time of mortality. Patients who were lost to follow-up or succumbed to causes unassociated with PDAC were treated as censored events. Kaplan-Meier estimator plots were constructed, and the differences between groups were analyzed using a log rank test. Univariate and multivariate Cox proportional hazards regression analysis was performed to investigate the association between the MIF expression or clinical characteristics of patients and overall survival. Significant prognostic factors identified using univariate analysis were further evaluated by multivariate Cox regression analysis. All statistical tests 
Table II. Univariate and multivariate analysis of factors associated with OS.

\begin{tabular}{|c|c|c|c|}
\hline Clinical variables & Case no. & $\operatorname{HR}(95 \% \mathrm{CI})^{\mathrm{a}}$ & P-value ${ }^{a}$ \\
\hline \multicolumn{4}{|l|}{ Univariate analysis } \\
\hline MIF (High vs. low) & $43 / 42$ & $1.748(1.059-2.888)$ & $0.029^{\mathrm{b}}$ \\
\hline Sex (Male vs. female) & $51 / 34$ & $1.039(0.628-1.729)$ & 0.883 \\
\hline Age ( $>50$ vs. $\leq 50$ years $)$ & $69 / 16$ & $1.372(0.696-2.706)$ & 0.361 \\
\hline Tumor size ( $>3 \mathrm{vs} . \leq 3 \mathrm{~cm})$ & $48 / 37$ & $1.541(0.724-2.687)$ & 0.125 \\
\hline Differentiation (Poor vs. well/moderate) & $52 / 33$ & $3.858(2.280-6.526)$ & $<0.001^{\mathrm{b}}$ \\
\hline TNM stage (III/IV vs. I/II) & $49 / 36$ & $2.001(1.401-3.061)$ & $<0.001^{\mathrm{b}}$ \\
\hline Lymphatic spread (Yes vs. no) & $36 / 49$ & $2.452(1.461-4.118)$ & $0.001^{\mathrm{b}}$ \\
\hline Hepatic metastasis (Yes vs. no) & $19 / 66$ & $2.200(1.236-3.916)$ & $0.007^{\mathrm{b}}$ \\
\hline Serum CEA ( $\geq 10$ vs. $<10$ ng/ml) & $49 / 36$ & $1.584(0.954-2.628)$ & 0.075 \\
\hline \multicolumn{4}{|l|}{ Multivariate analysis } \\
\hline MIF (High vs. low) ${ }^{\mathrm{a}}$ & $43 / 42$ & $1.916(1.140-3.218)$ & $0.014^{\mathrm{b}}$ \\
\hline TNM stage (III/IV vs. I/II) & $49 / 36$ & $2.706(1.550-4.723)$ & $<0.001^{\mathrm{b}}$ \\
\hline Differentiation (Poor vs. well/moderate) & $52 / 33$ & $3.552(2.051-6.151)$ & $<0.001^{\mathrm{b}}$ \\
\hline
\end{tabular}

Analysis was performed on 85 cases. The MIF level was examined by immunohistochemical staining. Median value of all 85 cases was chosen as the cut-off point for separating low MIF expression tumors from high MIF expression cases. ${ }^{\text {aHR }}$ (hazard ratio) and P-values were calculated using univariate or multivariate Cox proportional hazards regression; $95 \% \mathrm{CI}, 95 \%$ confidence interval. ${ }^{b} \mathrm{P}<0.05$, statistically significant difference. MIF, Macrophage migration inhibitory factor; OS, overall survival; TNM, tumor node metastasis; CEA, carcinoembryonic antigen; CI, confidence interval.

were two-tailed, and $\mathrm{P}<0.05$ was considered to indicate a statistically significant difference. Analyses were performed using SPSS software (version 19.0; IBM Corp., Armonk, NY, USA).

Data are expressed as the mean \pm standard error of the mean from three independent experiments. Differences between the groups were analyzed by student's t-test or one-way analysis of variance with Bonferroni's post hoc test. Analyses were performed with GraphPad Prism, version 5 (GraphPad Software, Inc., San Diego, CA, USA).

\section{Results}

Overexpression of MIF is a frequent event in PDAC tissue. It has been reported that MIF is overexpressed in pancreatic cancer $(11,18)$. To further confirm this, MIF expression was evaluated in 85 paired PDAC and adjacent noncancerous tissues by IHC. MIF expression was observed in 75/85 tumor samples (Fig. 1A). Compared with paired noncancerous tissue, the majority (75/85) of PDAC tumor tissue exhibited significantly higher MIF expression (Fig. 1B). Consistent with the results from IHC analysis, RT-sqPCR and immunoblotting assays revealed a similar trend with increased MIF expression in PDAC tissue at the mRNA and protein levels (Fig. 1C). These data indicate that MIF overexpression is prevalent in PDAC.

Increased MIF expression is associated with poor survival of patients with PDAC. Whether MIF overexpression is associated with clinical features or clinical outcome of patients with PDAC was investigated. MIF expression was divided into low- and high-expression groups, based on median expression (cut-off value, 1.08) analyzed by IHC in all PDAC cases The associations between the MIF expression and clinical features are summarized in Table I. Increased MIF expression was identified to be associated with advanced age, lymphatic spread and increased serum carcinoembryonic antigen levels (Table I). Furthermore, Kaplan-Meier analysis revealed that higher MIF levels were significantly associated with shorter overall survival time of patients with $\operatorname{PDAC}(\mathrm{P}=0.023$; Fig. 2). Cox proportional hazards regression analysis was performed to exclude confounder effects. Univariate Cox analysis was first performed to identify factors that may affect the overall survival of patients with PDAC. Higher MIF expression, poor differentiation, high tumor node metastasis (TNM) staging and the presence of metastasis were associated with inferior survival times (Table II). Multivariate Cox analysis adjusted for differentiation grade and TNM stage further confirmed that MIF overexpression was an independent risk factor for poor overall survival in patients with PDAC (Table II). These data suggest that overexpression of MIF may predict the poor survival and may contribute to PDAC metastasis.

Knockdown of MIF suppresses proliferation and invasion of PDAC cells. The association of MIF expression with clinical features and poor outcomes for patients with PDAC prompted an investigation into the potential role of MIF in PDAC growth and metastasis. Thus, the potential role of MIF in the regulation of G1/S transition and in vitro invasion of two PDAC cell lines, Panc-1 and Bxpc-3, was examined. Cells were transfected with si-MIF or with si-NC, and were then subjected to a FACS or Boyden chamber Transwell invasion assay. RT-sqPCR and immunoblotting assays demonstrated that si-MIF transfection markedly decreased the mRNA and 

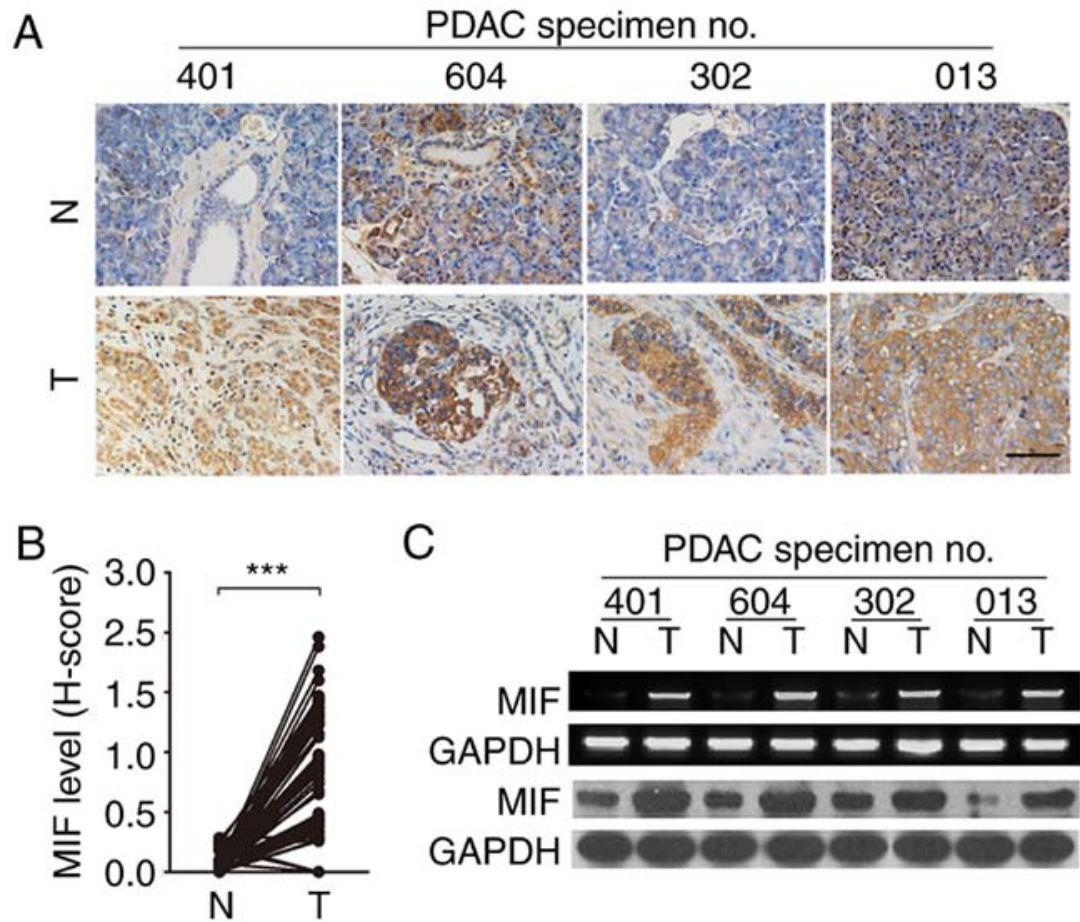

C

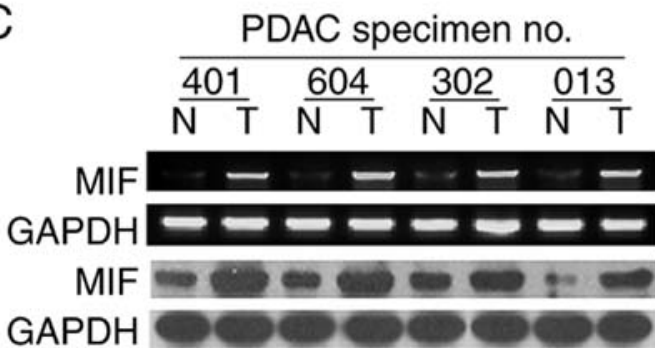

Figure 1. Analysis of MIF expression in paired PDAC and adjacent non-tumor tissues. (A) Immunohistochemistry analysis of MIF expression in 85 human PDAC samples. A brown signal was considered positive. Images were captured at $\mathrm{x} 400$ magnification. Scale bar, $50 \mu \mathrm{m}$. (B) Comparison of the MIF level in PDAC and adjacent non-tumor tissues in 85 human PDAC samples. Statistical analysis was performed by Wilcoxon matched-pairs signed-ranks test. (C) Reverse transcription-semi-quantitative polymerase chain reaction and immunoblotting analysis of MIF expression in PDAC. GAPDH was used as internal control. ${ }^{* * *} \mathrm{P}<0.001$. MIF, Macrophage migration inhibitory factor; PDAC, pancreatic ductal adenocarcinoma; T, PDAC tissue; $\mathrm{N}$, adjacent noncancerous tissue.

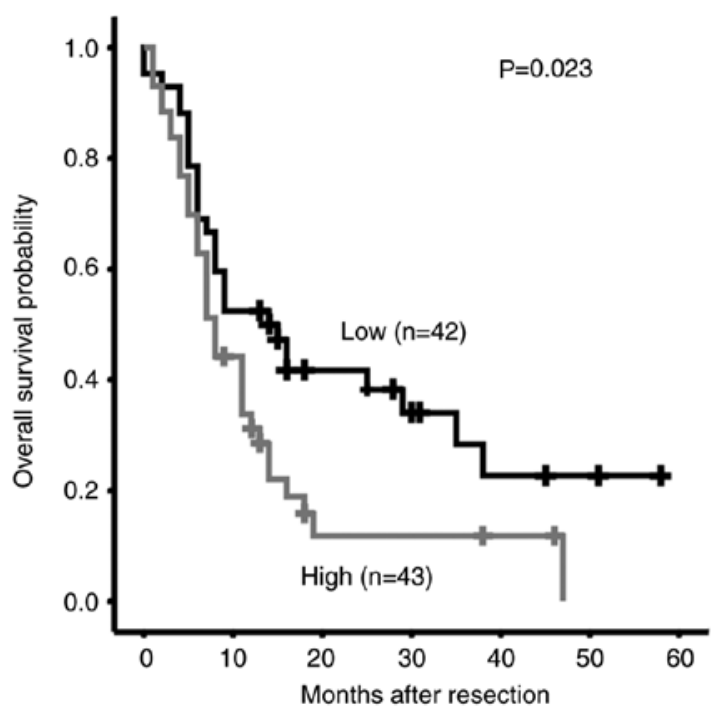

Figure 2. Kaplan-Meier analysis for pancreatic ductal adenocarcinoma patient survival according to MIF level. MIF levels were analyzed by immunohistochemistry, and the median value of all 85 cases was chosen as the cut-off point for separating MIF low-expression tumors $(n=42)$ from MIF high-expression cases ( $\mathrm{n}=43)$. MIF, Macrophage migration inhibitory factor.

protein levels of MIF in pancreatic cancer cells (Fig. 3A). Notably, the knockdown of MIF resulted in a marked accumulation of the G1-population in PDAC cells (Fig. 3B and C). Furthermore, si-MIF-transfected Panc-1 and Bxpc-3 cells exhibited a significantly reduced number of cells invading through the Transwell chamber (Fig. 3D and E).
Knockdown of MIF inhibits the activation of AKT and ERK, and suppresses the expression of cyclin D1 and MMP-2. The molecular mechanisms underlying the metastasis-promoting effects of MIF were investigated. The function of MIF is associated with two major tumor-promoting signaling pathways, namely the AKT and ERK signaling pathways $(9,10)$ Consistently, silencing MIF in PDAC cells significantly decreased the phosphorylation levels of AKT and ERK, but had no effect on the expression of total AKT and ERK protein (Fig. 4A and B), indicating that MIF knockdown inhibited the activation of AKT and ERK signaling in PDAC cells. Cyclin D1 and MMP-2 are important factors upregulated by AKT and ERK signaling that execute AKTand ERK-mediated cell invasion (19-22). The mRNA and protein levels of cyclin D1 and MMP-2 were significantly reduced in si-MIF-transfected cells compared with the NC group (Fig. 4C and D). These data suggest the MIF enhances PDAC metastasis by activating the AKT and ERK pathways, which in turn upregulates the expression of cyclin D1 and MMP-2.

\section{Discussion}

PDAC manifests as a highly aggressive cancer with poor prognosis. The identification of molecules that are involved in PDAC tumor progression and aggressiveness may elucidate novel targets for PDAC treatment. Previous studies have suggested that MIF, a pro-inflammatory cytokine, facilitates cancer progression, associating inflammation with pancreatic cancer progression (12-14,23). Emerging research has explored the biological effects of MIF in PDAC (11-14,23). Similar to 
A

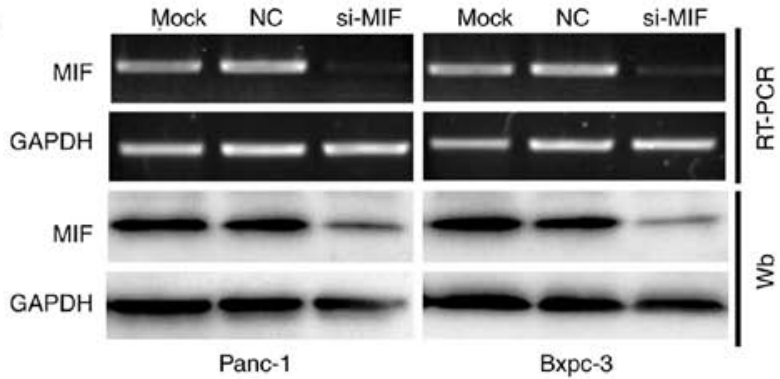

B

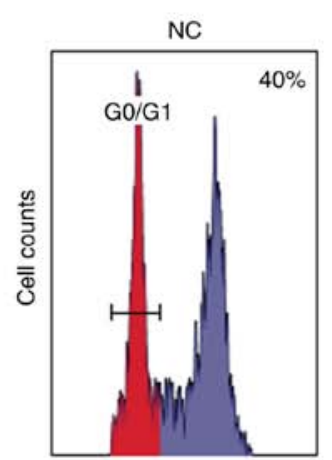

$2 \mathrm{~N} \quad 4 \mathrm{~N}$

Panc-1

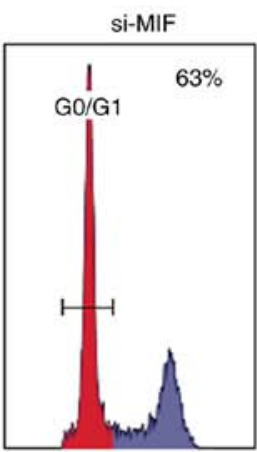

$2 \mathrm{~N} \quad 4 \mathrm{~N}$

D
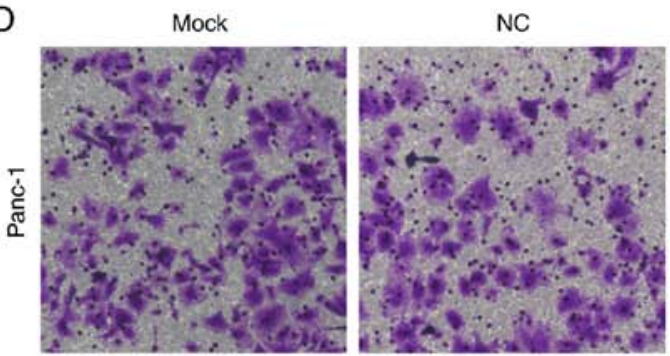

NC

$E$

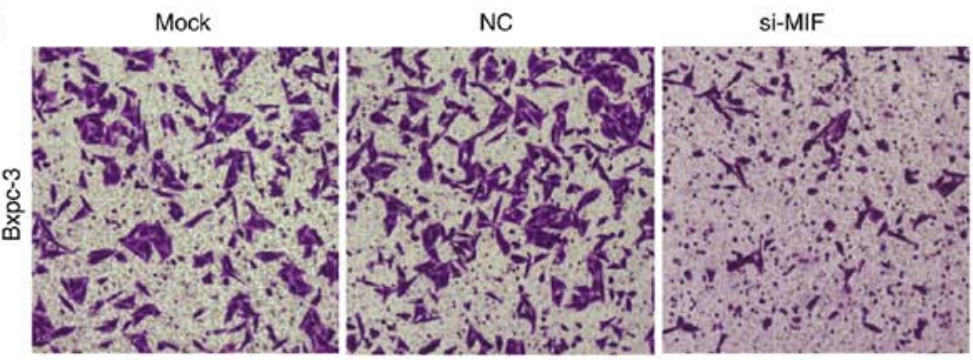

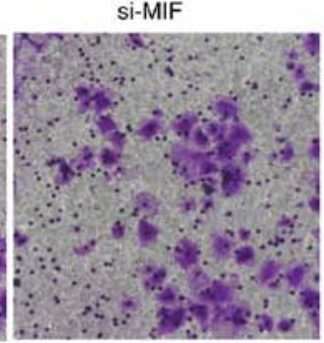

C

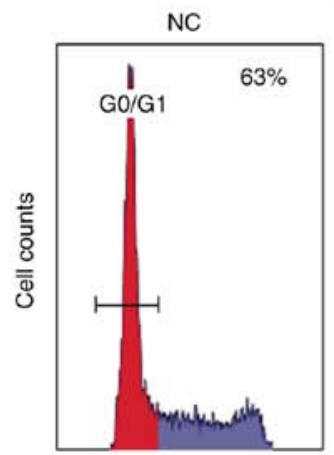

$2 \mathrm{~N} \quad 4 \mathrm{~N}$
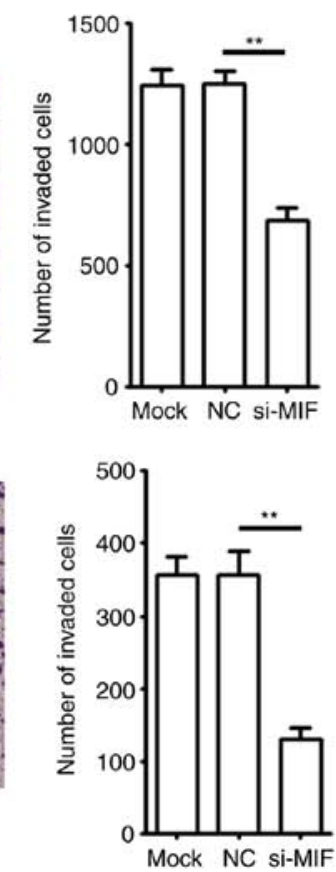

Figure 3. Silencing of MIF expression inhibits tumor invasion in vitro. (A) siRNA-mediated knockdown of MIF in PDAC cells. Control Panc-1 or Bxpc-3 cells (Mock) and Panc-1 or Bxpc-3 cells transfected with $50 \mathrm{nM}$ MIF siRNA (si-MIF) or NC were subjected to RT-sqPCR or immunoblotting analysis. GAPDH was used as internal control. MIF knockdown caused accumulation of G1-population. (B) Panc-1 or (C) Bxpc-3 cells transfected with NC or si-MIF were treated with nocodazole $(40 \mathrm{ng} / \mathrm{ml}) 32 \mathrm{~h}$ after transfection, and then cultured for an additional $16 \mathrm{~h}$ before harvest for FACS analysis. The percentage of the G1-population is indicated within each histogram. The results were reproduced in two independent experiments and representative data are shown. MIF knockdown inhibited PDAC (D) Panc-1 or (E) Bxpc-3 cell invasion. Control Panc-1 or Bxpc-3 cells (Mock) and Panc-1 or Bxpc-3 cells transfected with NC or si-MIF were added to Transwell chambers with Matrigel coating and incubated for $24 \mathrm{~h}$, followed by staining with crystal violet. Scale bar, $100 \mu \mathrm{m}$. ${ }^{* *} \mathrm{P}<0.01$. MIF, Macrophage migration inhibitory factor; PDAC, pancreatic ductal adenocarcinoma; si/siRNA, small inteferring RNA; NC, negative control siRNA; wb, western blot; RT-sqPCR, reverse transcription-semi-quantitative polymerase chain reaction.

other reports that have described a regulatory function of MIF in cell growth and survival (24-29), pro-proliferation and anti-apoptosis roles for MIF in PDAC have been identified (11). Furthermore, it has been reported that MIF induces epithelial to mesenchymal transition and enhances tumor aggressiveness in PDAC $(13,14)$. Additionally, exosome-derived MIF may prime the liver for PDAC metastasis, and may be a potential biomarker for liver metastasis (23). Consistently, a pro-metastasis effect was observed regarding MIF in PDAC in present study. Taken together, these data highlight the importance 
A

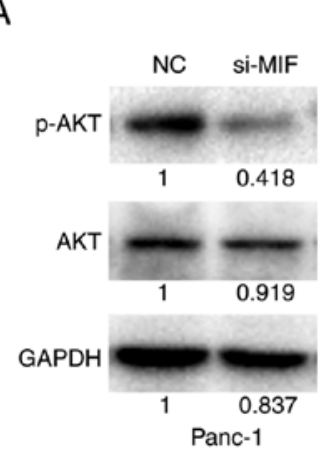

B

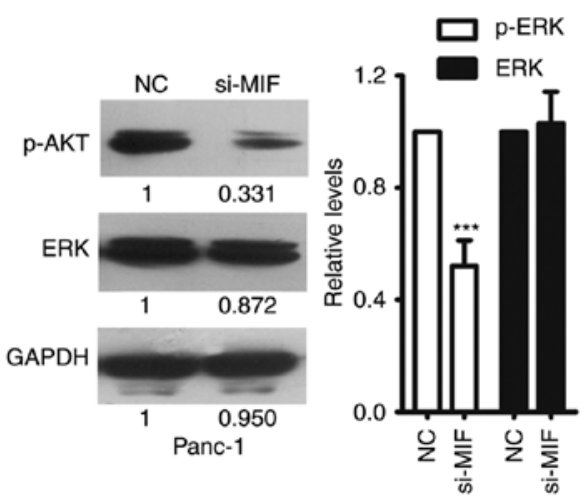

C

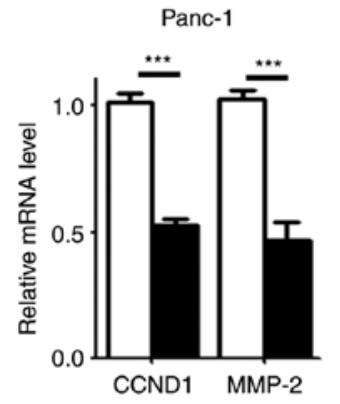

D

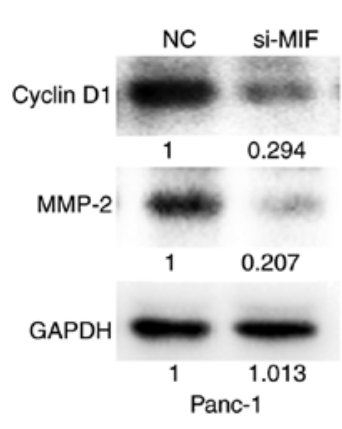

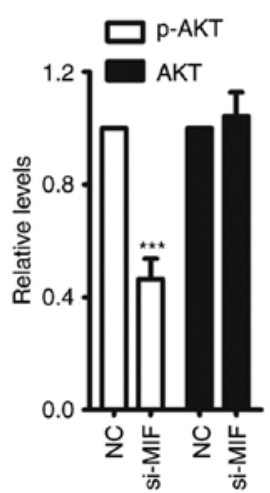

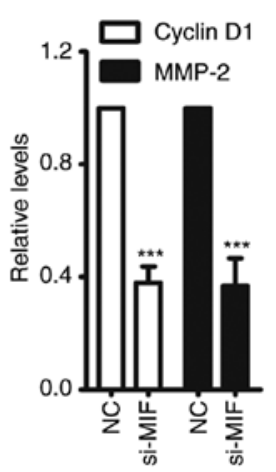

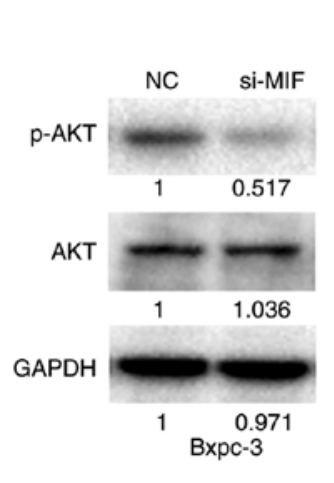
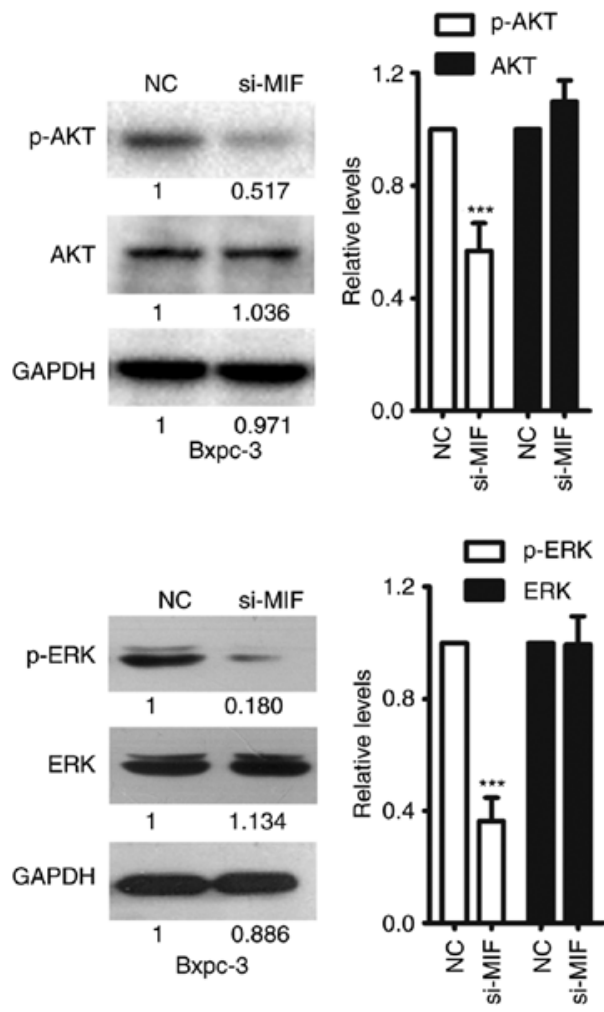

Bxpc-3
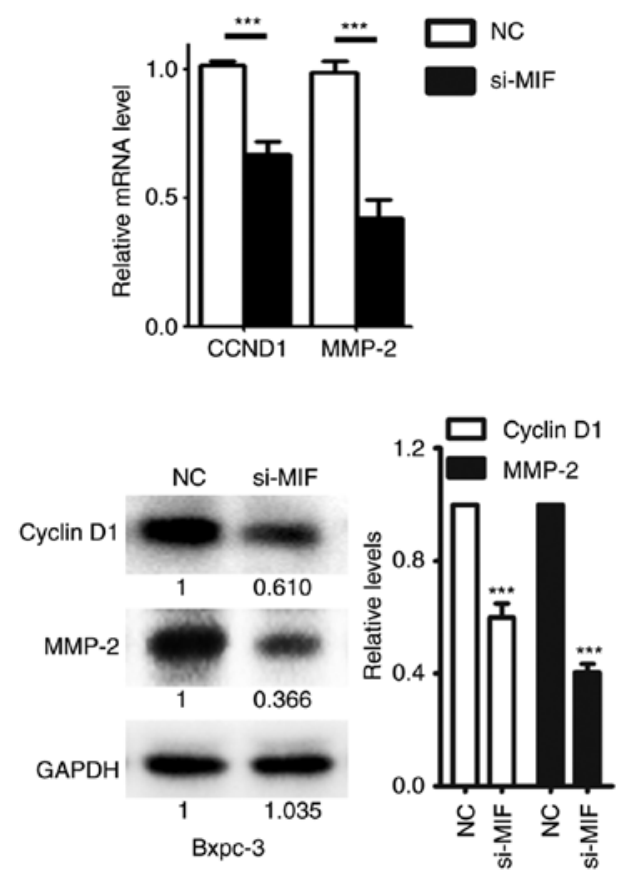

Figure 4. Silencing of MIF expression negatively regulates the AKT and ERK signaling pathways. Knockdown of MIF attenuated AKT and ERK activities in PDAC cells. Panc-1 or Bxpc-3 cells were transfected with NC or si-MIF and subjected to immunoblotting for (A) p-AKT and AKT; and (B) p-ERK1/2 and ERK1/2 expression. GAPDH was used as an internal control. Knockdown of MIF decreased the mRNA and protein levels of CCND1 and MMP-2. Panc-1 or Bxpc-3 cells transfected with si-MIF or NC were subjected to (C) reverse transcription- quantitative polymerase chain reaction or (D) immunoblotting analysis of CCND1 and MMP-2. For (A), (B), and (D), the intensity of each band was densitometrically quantified using Image J software and was normalized according to the value of $\mathrm{NC}$ group. Results were reproduced in three independent experiments and the cumulative data as well as the representative immunoblots are shown. ${ }^{* * *} \mathrm{P}<0.001$. MIF, Macrophage migration inhibitory factor; PDAC, pancreatic ductal adenocarcinoma; si/siRNA, small inteferring RNA; NC, negative control siRNA; CCND1, cyclin D1; MMP-2, matrix metalloproteinase-2; p- phosphorylated; AKT, AKT serine/threonine kinase; ERK, extracellular-signal-regulated kinase.

of MIF overexpression in promoting PDAC progression, and suggest that inhibition of MIF may offer a novel therapeutic option for treatment of PDAC.
Although MIF overexpression has been observed in patients with PDAC, to the best of our knowledge, only one report has explored the associations between MIF expression, 
disease aggressiveness and clinical outcome (14). To date, only one other group has indicated an association between increased tumor expression of MIF and decreased survival rates in patients with PDAC following tumor resection (14). In the present study, the overexpression of MIF was demonstrated to be a frequent event in PDAC. Notably, the peritumorial tissues of PDAC expressed undetectable or low levels of MIF protein in acinar and ductal cells as well as stromal cells. As known, PDAC is associated with intra- and peritumorial inflammation, which may significantly induce MIF expression in peritumorial tissues (30). The variation in MIF expression between the peritumorial tissues of PDAC samples may be at least partly due to the differing degree of peritumorial inflammation. The results of the present study demonstrated that high levels of MIF were associated with metastasis and inferior survival of patients with PDAC, and that high MIF expression may serve as an independent risk factor for poor disease outcome. Using an siRNA-based strategy, the pro-metastasis effect of endogenous MIF expression was examined in human PDAC cell lines, further supporting the role for MIF in PDAC aggressiveness.

PDAC has a high tendency to metastasize. Typically, PDAC cells first spread to nearby lymph nodes, and later metastasize to the liver and other organs. ${ }^{2}$ Thus, lymphatic spread is a critical early event of PDAC progression. Consistently, the present study demonstrated that lymphatic spread and hepatic metastasis are significantly associated with poor survival of patients with PDAC. The association between high MIF expression and lymphatic spread indicated that MIF may be a possible mediator of extensive lymph node metastasis. Furthermore, the finding that silencing of MIF inhibits the invasion of PDAC cells in vitro supports the possibility that MIF facilitates lymph node metastasis of PDAC cells. Nevertheless, this hypothesis requires further evaluation using additional in vivo studies.

The present study results revealed that that silencing of MIF significantly inhibited the expression of MMP-2 and CCND1, suggesting that MMP-2 and CCND1 may be target genes that mediate the pro-metastasis effects of MIF. MMP-2 is a member of the matrix metalloproteinase family, is frequently overexpressed in tumors, and is well known to facilitate invasion and metastasis of tumor cells by degrading extracellular matrix (31). CCND1, a regulator of cell cycle $(32,33)$, promotes tumor invasion and metastasis, according to evidence from clinical studies and in vivo experiments $(34,35)$. On one hand, nuclear CCND1 and its binding partner $\mathrm{Cdk} 4$ act as a transcriptional regulator of genes controlling cell adherence and migration (36,37). On the other hand, CCND1 in the cytoplasm phosphorylates cytoplasmic and membrane-associated proteins to promote cell spreading and invasion (38). Notably, we previously reported that MIF promotes hepatocellular carcinoma cell growth by positively regulating CCND1 expression (26). The data from cell cycle analysis also revealed that silencing of MIF blocked G1/S transition of PDAC cells (data not shown), indicating that MIF promoted PDAC cell cycle by inducing CCND1 expression. Taken together with the results of the present study, these data indicate that CCND1 may serve a central role in MIF-mediated tumor progression.

In conclusion, the findings of the current study identified a MIF/AKT/ERK/CCND1/MMP-2 cascade that promotes
PDAC metastasis. The results suggest that MIF is a candidate prognostic indicator in patients following resection of PDAC. Additional pre-clinical studies are necessary to evaluate the effect of MIF-targeting in pancreatic cancer as a novel therapeutic approach.

\section{Acknowledgements}

Not applicable.

\section{Funding}

The present study was supported by grants from the National Natural Science Foundation of China (grant nos. 81672417, 81370368,81172337 and 81502464), and the Science and Technology Foundation of Guangdong Province grant (grant nos. 2014A020212647 and 2014A020212083).

\section{Availability of data and materials}

All data generated or analyzed during this study are included in this published article.

\section{Authors' contributions}

WL and JTX designed the study. DW, RW, AH and ZF performed the experiments. DW, KW and $\mathrm{MH}$ analyzed the experimental data. $\mathrm{AH}$ and $\mathrm{MH}$ collected the tissue specimens. WL, JTX, DW and RW wrote the manuscript. All authors discussed the results and contributed to the manuscript. All authors read and approved the final manuscript.

\section{Ethics approval and consent to participate}

The present study was approved by the Human Research Ethics Committees of the First Affiliated Hospital of Sun Yat-sen University (approval no. 201515; Guangzhou, China), according to the Declaration of Helsinki. All of patients enrolled in the present study provided written informed consent.

\section{Patient consent for publication}

Not applicable.

\section{Competing interests}

The authors declare that they have no competing interests.

\section{References}

1. Siegel RL, Miller KD and Jemal A: Cancer statistics, 2015. CA Cancer J Clin 65: 5-29, 2015.

2. Wolfgang CL, Herman JM, Laheru DA, Klein AP, Erdek MA, Fishman EK and Hruban RH: Recent progress in pancreatic cancer. CA Cancer J Clin 63: 318-348, 2013.

3. Liu SL, Friess H, Kleeff J, Ji ZL and Buchler MW: Surgical approaches for resection of pancreatic cancer: An overview. Hepatobiliary Pancreat Dis Int 1: 118-125, 2002.

4. Ryan DP, Hong TS and Bardeesy N: Pancreatic adenocarcinoma. N Engl J Med 371: 2140-2141, 2014.

5. Garcea G, Dennison AR, Steward WP and Berry DP: Role of inflammation in pancreatic carcinogenesis and the implications for future therapy. Pancreatology 5: 514-529, 2005 
6. Hausmann S, Kong B, Michalski C, Erkan M and Friess H: The role of inflammation in pancreatic cancer. Adv Exp Med Biol 816: 129-151, 2014

7. Gukovsky I, Li N, Todoric J, Gukovskaya A and Karin M: Inflammation, autophagy, and obesity: Common features in the pathogenesis of pancreatitis and pancreatic cancer. Gastroenterology 144: 1199-1209 e1194, 2013.

8. Lippitz BE: Cytokine patterns in patients with cancer: A systematic review. Lancet Oncol 14: e218-e228, 2013.

9. Schwartz V, Lue H, Kraemer S, Korbiel J, Krohn R, Ohl K, Bucala R, Weber C and Bernhagen J: A functional heteromeric MIF receptor formed by CD74 and CXCR4. FEBS Lett 583: 2749-2757, 2009

10. Leng L, Metz CN, Fang Y, Xu J, Donnelly S, Baugh J, Delohery T, Chen Y, Mitchell RA and Bucala R: MIF signal transduction initiated by binding to CD74. J Exp Med 197: 1467-1476, 2003.

11. Denz A, Pilarsky C, Muth D, Ruckert F, Saeger HD and Grutzmann R: Inhibition of MIF leads to cell cycle arrest and apoptosis in pancreatic cancer cells. J Surg Res 160: 29-34, 2010.

12. Guo D, Guo J, Yao J, Jiang K, Hu J, Wang B, Liu H, Lin L, Sun W and Jiang X: D-dopachrome tautomerase is over-expressed in pancreatic ductal adenocarcinoma and acts cooperatively with macrophage migration inhibitory factor to promote cancer growth. Int J Cancer 139: 2056-2067, 2016.

13. Yang S, He P, Wang J, Schetter A, Tang W, Funamizu N, Yanaga K, Uwagawa T, Satoskar AR, Gaedcke J, et al: A novel MIF signaling pathway drives the malignant character of pancreatic cancer by targeting NR3C2. Cancer Res 76: 3838-3850, 2016.

14. Funamizu N, Hu C, Lacy C, Schetter A, Zhang G, He P, Gaedcke J, Ghadimi MB, Ried T, Yfantis HG, et al: Macrophage migration inhibitory factor induces epithelial to mesenchymal transition, enhances tumor aggressiveness and predicts clinical outcome in resected pancreatic ductal adenocarcinoma. Int J Cancer 132: 785-794, 2013

15. Klöppel G, Hruban RH, DS, Longnecker DS, Adler G, Kern SE and Partanen TJ: Ductal adenocarcinoma of the pancreas. In: Pathology and Genetics of Tumours of the Digestive System. Hamilton SR and Aaltonen LA (eds). International Agency for Research on Cancer Press, Lyon, France, pp221-230, 2000.

16. Wang R, Zhao N, Li S, Fang JH, Chen MX, Yang J, Jia WH, Yuan Y and Zhuang SM: MicroRNA-195 suppresses angiogenesis and metastasis of hepatocellular carcinoma by inhibiting the expression of VEGF, VAV2, and CDC42. Hepatology 58: 642-653, 2013.

17. Livak KJ and Schmittgen TD: Analysis of relative gene expression data using real-time quantitative PCR and the $2^{-\Delta \Delta C_{\mathrm{T}}}$ method. Methods 25: 402-408, 2001.

18. Tan L, Ye X, Zhou Y, Yu M, Fu Z, Chen R, Zhuang B, Zeng B, Ye $\mathrm{H}, \mathrm{Gao} \mathrm{W}$, et al: Macrophage migration inhibitory factor is overexpressed in pancreatic cancer tissues and impairs insulin secretion function of $\beta$-cell. J Transl Med 12: 92, 2014

19. Lauring J,Park BH and Wolff AC: The phosphoinositide-3-kinaseAkt-mTOR pathway as a therapeutic target in breast cancer. J Natl Compr Canc Netw 11: 670-678, 2013.

20. Cohen P and Frame S: The renaissance of GSK3. Nat Rev Mol Cell Biol 2: 769-776, 2001.

21. Liu J, Ben QW, Yao WY, Zhang JJ, Chen DF, He XY, Li L and Yuan YZ: BMP2 induces PANC-1 cell invasion by MMP-2 overexpression through ROS and ERK. Front Biosci 17: 2541-2549, 2012.

22. Dong QZ, Wang Y, Tang ZP, Fu L, Li QC, Wang ED and Wang EH: Derlin-1 is overexpressed in non-small cell lung cancer and promotes cancer cell invasion via EGFR-ERK-mediated up-regulation of MMP-2 and MMP-9. Am J Pathol 182: 954-964, 2013.
23. Costa-Silva B, Aiello NM, Ocean AJ, Singh S, Zhang H, Thakur BK, Becker A, Hoshino A, Mark MT, Molina H, et al: Pancreatic cancer exosomes initiate pre-metastatic niche formation in the liver. Nat Cell Biol 17: 816-826, 2015.

24. Richard V, Kindt N, Decaestecker C, Gabius HJ, Laurent G, Noel JC and Saussez S: Involvement of macrophage migration inhibitory factor and its receptor (CD74) in human breast cancer. Oncol Rep 32: 523-529, 2014

25. Oliveira CS, de Bock CE, Molloy TJ, Sadeqzadeh E, Geng XY, Hersey P, Zhang XD and Thorne RF: Macrophage migration inhibitory factor engages $\mathrm{PI} 3 \mathrm{~K} / \mathrm{Akt}$ signalling and is a prognostic factor in metastatic melanoma. BMC Cancer 14: 630, 2014.

26. Huang XH, Jian WH, Wu ZF, Zhao J, Wang H, Li W and Xia JT: Small interfering RNA (siRNA)-mediated knockdown of macrophage migration inhibitory factor (MIF) suppressed cyclin D1 expression and hepatocellular carcinoma cell proliferation. Oncotarget 5: 5570-5580, 2014.

27. Wang C, Zhou X, Li W, Li M, Tu T, Ba X, Wu Y, Huang Z, Fan G, Zhou G, et al: Macrophage migration inhibitory factor promotes osteosarcoma growth and lung metastasis through activating the RAS/MAPK pathway. Cancer Lett 403: 271-279, 2017.

28. Bifulco C, McDaniel K, Leng L and Bucala R: Tumor grow th-promoting properties of macrophage migration inhibitory factor. Curr Pharm Des 14: 3790-3801, 2008

29. Lue H, Thiele M, Franz J, Dahl E, Speckgens S, Leng L, Fingerle-Rowson G, Bucala R, Lüscher B and Bernhagen J: Macrophage migration inhibitory factor (MIF) promotes cell survival by activation of the Akt pathway and role for CSN5/JAB1 in the control of autocrine MIF activity. Oncogene 26: 5046-5059, 2007.

30. Candido J and Hagemann T: Cancer-related inflammation. J Clin Immunol 1 (Suppl 33): S79-S84, 2013.

31. Kessenbrock K, Plaks V and Werb Z: Matrix metalloproteinases: Regulators of the tumor microenvironment. Cell 141: 52-67, 2010.

32. Sherr CJ and Roberts JM: Living with or without cyclins and cyclin-dependent kinases. Genes Dev 18: 2699-2711, 2004.

33. Bienvenu F, Jirawatnotai S, Elias JE, Meyer CA, Mizeracka K, Marson A, Frampton GM, Cole MF, Odom DT, Odajima J, et al: Transcriptional role of cyclin D1 in development revealed by a genetic-proteomic screen. Nature 463: 374-378, 2010.

34. Drobnjak M, Osman I, Scher HI, Fazzari M and Cordon-Cardo C: Overexpression of cyclin D1 is associated with metastatic prostate cancer to bone. Clin Cancer Res 6: 1891-1895, 2000.

35. Huang $\mathrm{H}, \mathrm{Hu} \mathrm{YD}, \mathrm{Li} \mathrm{N}$ and Zhu Y: Inhibition of tumor growth and metastasis by non-small cell lung cancer cells transfected with cyclin D1-targeted siRNA. Oligonucleotides 19: 151-162, 2009.

36. Li Z, Jiao X, Wang C, Ju X, Lu Y, Yuan L, Lisanti MP, Katiyar S and Pestell RG: Cyclin D1 induction of cellular migration requires p27 ${ }^{\mathrm{KIP} 1}$. Cancer Res 66: 9986-9994, 2006.

37. Li Z, Wang C, Jiao X, Lu Y, Fu M, Quong AA, Dye C, Yang J, Dai M, Ju X, et al: Cyclin D1 regulates cellular migration through the inhibition of thrombospondin 1 and ROCK signaling. Mol Cell Biol 26: 4240-4256, 2006.

38. Fuste NP, Fernandez-Hernandez R, Cemeli T, Mirantes C, Pedraza N, Rafel M, Torres-Rosell J, Colomina N, Ferrezuelo F, Dolcet X, et al: Cytoplasmic cyclin D1 regulates cell invasion and metastasis through the phosphorylation of paxillin. Nat Commun 7: 11581, 2016.

This work is licensed under a Creative Commons Attribution-NonCommercial-NoDerivatives 4.0 International (CC BY-NC-ND 4.0) License. 